\title{
Il personaggio delle Grandi Parodie Disney tra intertestualità e serialità
}

\author{
Giovanni Vito Distefano \\ Università degli Studi di Cagliari \\ gianvito.distefano@gmail.com
}

\begin{abstract}
Le Grandi Parodie Disney dei classici della letteratura costituiscono un'originale e intensa impresa creativa, cominciata nel 1949 con L'Inferno di Topolino e ancora oggi portata avanti con successo dagli autori Disney italiani. L'articolo si concentra sui procedimenti adattivi alla base delle Parodie, privilegiando quelli che investono in prima battuta l'elemento narrativo del personaggio. Il close reading condotto sull'Inferno di Topolino, parodia antesignana e manifesto dell'intero corpus, permette di osservare come sul personaggio della parodia si giochi in larga misura il mix di intertestualità e serialità che, con una considerevole prevalenza della seconda sulla prima, caratterizza questi adattamenti. In conclusione, si mostra come le risorse espressive e semantiche del personaggio seriale non solo comportino l'immediata riconoscibilità della parodia, ma sostengano significative innovazioni di ordine tematico-ideologico rispetto all'opera dantesca e coraggiose prese di posizione sull'importanza del fumetto seriale nel sistema culturale contemporaneo.
\end{abstract}

Parole chiave: Grandi Parodie Disney. personaggio seriale. Intertestualità. Divina Commedia. Topolino.

Resumo: Le Grandi Parodie Disney dos clássicos da literatura constituem um trabalho criativo original e intenso, que começou em 1949 com o Inferno de Mickey e ainda hoje é apresentado com sucesso por autores italianos da Disney. $\mathrm{O}$ artigo enfoca os procedimentos adaptativos subjacentes às paródias, privilegiando aquelas que envolvem, principalmente, o elemento narrativo do personagem. A leitura atenta realizada em O Inferno de Mickey, precursor da paródia e presente em todo o corpus, permite-nos observar como a mistura de intertextualidade e serialidade desempenha, em grande medida, o caráter da paródia que, com uma prevalência considerável do segundo sobre o primeiro, caracteriza essas adaptações. Desse modo, observa-se como os recursos expressivos e semânticos do personagem serial não só levam ao reconhecimento imediato da paródia, mas também sustentam significativas inovações temático-ideológicas no que diz respeito à obra de Dante e às posturas corajosas sobre a importância dos quadrinhos seriados no sistema cultural contemporâneo.

Palavras-chave: Grandi Parodie Disney. Personagem serial. Intertextualidade. Divina Commedia. Mickey Mouse. 
ABSTRACT: The Great Disney Parodies of the classics of literature are a major creative enterprise, begun in 1949 with L'Inferno di Topolino and still successfully carried out by Italian Disney authors today. The article investigates the adaptive procedures of the Parodies, with a special focus on those involving the pivotal narrative element of the character. From the close reading of L'Inferno di Topolino, the first parody to be written and a manifesto of the entire corpus, it is possible to observe how the mix of intertextuality and seriality which distinguishes these adaptations hinges largely on the expressive and semantic resources of Disney serial characters. In the conclusion, the main effects of the prevailing of seriality over intertextuality are discussed, showing how it makes for the immediate recognizability of the parody, supports significant thematic-ideological innovations with respect to Dante's work, and allows for courageous metaliterary assertion on the importance of serial comics in the contemporary cultural system.

Keywords: Grandi Parodie Disney. Serial character. Intertextuality. Divine Comedy. Mickey Mouse.

\section{Introduzione}

Il procedimento adattivo alla base delle Grandi Parodie Disney dei grandi classici della letteratura coinvolge in una coerente strategia di creazione derivativa tutti gli elementi del racconto: il tempo e lo spazio, lo stile e il genere narrativo, $\mathrm{i}$ temi e i personaggi ${ }^{1}$. Tuttavia, come in tutti i prodotti del brand disneyano, anche nel caso delle parodie, è una in particolare di queste dimensioni a dimostrare, se non una maggiore importanza, una più immediata visibilità. Sul sistema dei personaggi, più che su ogni altro elemento del racconto, si concentrano gli elementi distintivi dell'universo disneyano ed è esso a sostenere e a rendere possibile il processo di adattamento di un testo letterario canonico in una storia di Topi e Paperi.

In questo contributo si tenterà di tracciare alcune delle principali strategie adattive attive nelle Grandi Parodie Disney dei classici della lettura, assumendo la categoria narratologica del personaggio come ambito specifico di osservazione. Allo stesso tempo, dovendo scegliere un riferimento testuale sul quale esemplificare e sostenere le nostre considerazioni, sarà anche l'occasione per 
introdurre e commentare da vicino l'antenata di tutte le parodie, ovvero L'Inferno di Topolino.

1. Prima di aprire il testo e approfondire l'osservazione dell'adattamento, è opportuno premettere una breve introduzione di ordine generale, utile a chiarire pochi essenziali punti sulle peculiarità strutturali dei personaggi delle Parodie. Topolino, così come gli altri personaggi Disney, non è 'semplicemente' un topo antropomorfo, come sono i personaggi delle favole o quelli di un canone della parodia come la Batracomiomachia pseudo-omerica. Topolino, e con lui i più caratterizzati e noti dei personaggi Disney, è invece un mito contemporaneo e un personaggio seriale, protagonista di una lunghissima serie di storie. Lasciando il primo aspetto agli studi che coraggiosamente si inoltrano per lo scivoloso terreno della ricezione culturale dei personaggi Disney ${ }^{2}$, mi concentrerò invece sulla condizione, più oggettiva e almeno in apparenza puramente formale, della serialità.

Serialità significa, in breve, l'esistenza di un rapporto di ripetitività che accomuna storie diverse, appartenenti appunto a una stessa serie. Essa prevede, da una parte, un nucleo comune costante in tutti gli episodi, dall'altra, un certo grado di variabilità tra un episodio e l'altro. A rimanere invariato può essere, in diverse combinazioni e proporzioni, l'uno o l'altro degli elementi del racconto, i protagonisti, ad esempio, oppure i temi; possono condividere uno stesso filo narrativo suddiviso in più segmenti oppure avere in comune uno schema narrativo iterativo, ripetuto senza sostanziali modifiche di episodio in episodio $^{3}$. Una serie di successo trova modi efficaci di conciliare i due aspetti, quello dell'innovazione che rende distinti e unici i diversi episodi, e quello della ripetizione, che li accomuna e li rende riconoscibili. Tipico delle storie a fumetti Disney è l'ultima modalità indicata, ovvero il format delle serie 'senza memoria': in ogni singolo episodio si riattiva dal principio lo stesso ricorrente 
schema narrativo, con variazioni che non ne intaccano la sostanza. Per capirci, nella conclusione di un racconto di Topolino, il protagonista finirà sempre per sventare il progetto criminoso di Gambadilegno o per risolvere l'enigma, per poi ritrovarsi nella stessa situazione di partenza all'inizio dell'episodio successivo, senza che negli episodi a venire rimanga memoria di ciò che avviene nei precedenti.

Anche le Grandi Parodie Disney, pur eccezionali sotto altri punti di vista in quanto adattamenti intersemiotici di opere letterarie, sono a tutti gli effetti anelli della lunga catena delle storie di Topolino o Paperino. Da ciò deriva il carattere essenziale di queste storie a fumetti, la cui natura di adattamenti ha nel passaggio di medium solo uno, e non il principale, dei suoi ingredienti. Per comprendere il procedimento creativo alla base delle parodie Disney può essere utile accostare la serialità disneyana a quella tipica del cinema hollywoodiano ai tempi dello star system. In un suo studio, ormai storico, Umberto Eco distingueva tra le altre questa forma di serialità in quanto:

\begin{abstract}
Determinata dalla natura stessa dell'attore: la sola presenza di John Wayne, in assenza di regia molto personalizzata, non può che produrre lo stesso film, perché gli eventi nascono dalla mimica, dagli schemi comportamentali, talora dalla elementarità stessa del personaggio-attore, il quale non può che fare sempre e comunque le stesse cose. In questi casi, per quanto l'autore si ingegni a inventare storie diverse, di fatto il pubblico riconosce (con soddisfazione) sempre e comunque la stessa storia. (ECO 1985, p. 130).
\end{abstract}

Al modo in cui un grande divo del cinema interpreta la sua parte senza smettere per un istante di essere riconoscibile, producendo l'effetto finale indicato da Eco, i personaggi Disney danno corpo e voce ai protagonisti delle opere letterarie rimanendo al contempo se stessi, con tutti (o quasi) i tratti e i caratteri sviluppati nel corso della loro lunga esistenza di personaggi seriali.

Sono spesso gli stessi testi delle Parodie Disney a suggerire, metaletterariamente, la metafora drammaturgia quale paradigma partico- 
larmente idoneo per alludere al mix di serialità e intertestualità di questo meccanismo. Una rappresentazione drammatica 'in costume' da parte dei personaggi Disney è infatti una delle cornici narrative più frequenti nel corpus delle parodie $^{4}$. Essa ricorre peraltro anche ne L'Inferno di Topolino, che ora, concluse le premesse, possiamo finalmente aprire.

2. Disegnato da Angelo Bioletto e scritto da Guido Martina - il più auto-

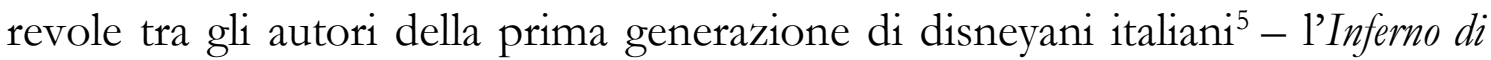
Topolino è stato pubblicato tra il 1949 e il 1950 nei nn. 7-12 di Topolino. Si noti la numerazione bassissima di Topolino a riprova di quanto importante e primordiale sia stato nella storia produttiva della Disney Italia il ricorso alle parodie. L'Inferno di Topolino è il primo esperimento di parodia letteraria, ma è già un indiscusso capolavoro ${ }^{6}$.

Lo è sul piano verbale, per la sua pregevole agilità linguistica, capace di divagare vivacemente nei registri del grottesco e del ludico, pur mantenendo costantemente un'eco dell'intonazione dantesca; sia nei dialoghi dei personaggi sia nel testo in terzine che accompagna, ininterrotto, tutta la storia. Lo è sul piano narrativo, per la capacità di preservare, pur innovandolo, lo schema diegetico del viaggio nell'oltretomba. Lo è sul piano tematico, sia quando ripropone in modo fedele la condizione di alcuni dannati danteschi, sia quando, più spesso, coglie il significato profondo del principio del contrappasso per disegnare, con procedimento analogo, un affresco della società e dei costumi attuali.

Nell'incipit della storia (Fig. 1) si trova la cornice drammaturgica della quale si è già fatto cenno. La introduce da subito il lungo cartello posto subito dopo il titolo:

Correva l'anno tal dei tali, e l'orologio della torre suonava le dieci battendo venti rintocchi perché era balbuziente. In quel momento Topolino e Pippo mietevano applausi recitando una grande commedia, anzi una Divina Commedia, nella quale Topolino faceva la parte di 
Dante, nientemeno... e Pippo, naturalmente, faceva Virgilio, nientepopodimeno! [...].

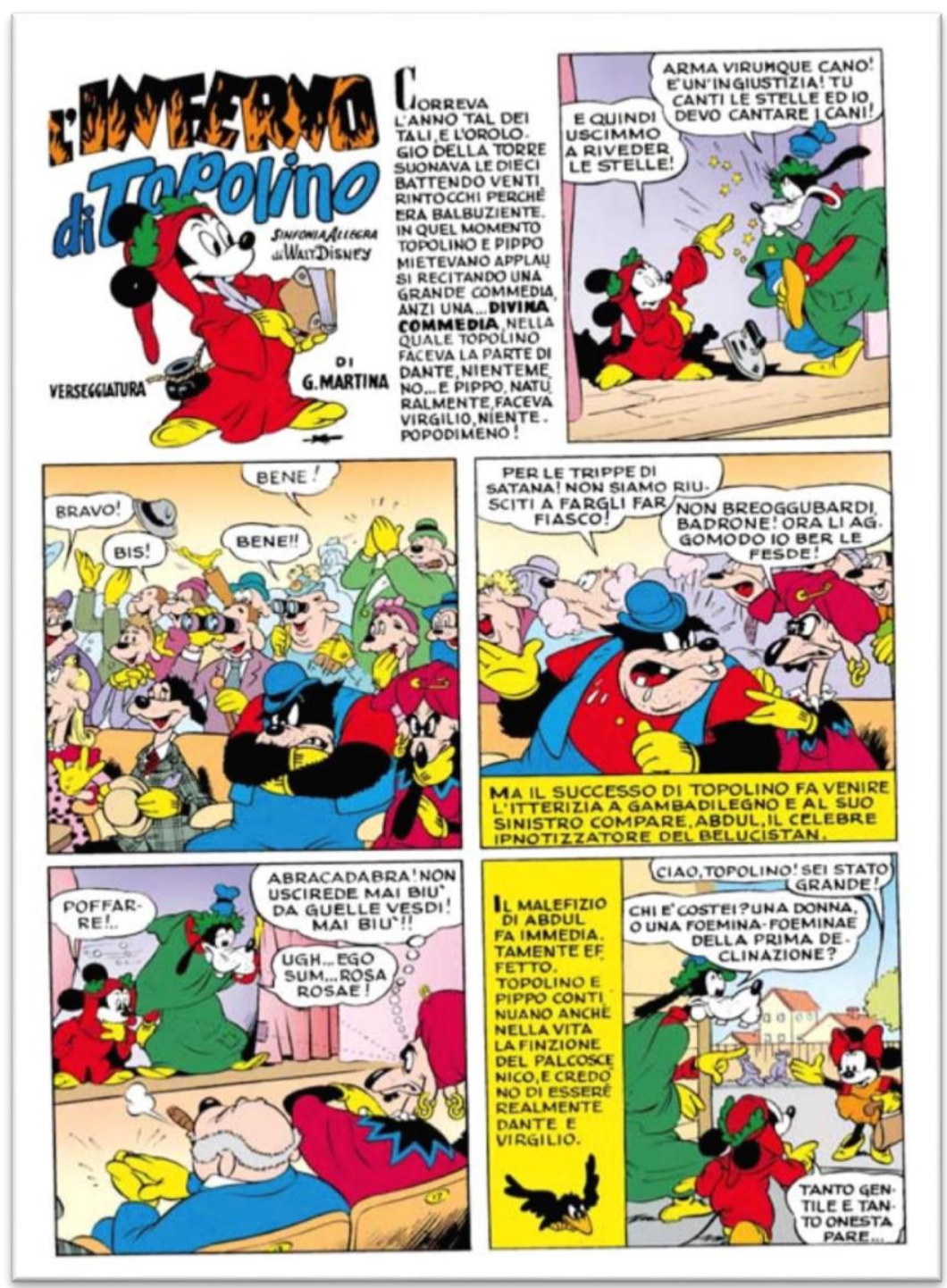

Figura 1

Dopo la divertita indicazione temporale, il testo racconta la messa in scena teatrale realizzata dai due protagonisti e procede immediatamente a indicare la distribuzione delle parti principali ("Topolino faceva [...] e Pippo, naturalmente, faceva...”). En passant si permette inoltre, sempre entro la sfera semantica del teatro, il gioco di parole tra il titolo del poema, da sempre oggetto di dibattito tra gli studiosi, e il nome della forma teatrale, la commedia appunto. 
La prima vignetta, contigua al cartello, visualizza il contenuto narrativo del cartello di testo e raffigura Topolino e Pippo travestiti da Dante e Virgilio, mentre concludono la loro strampalata declamazione dell'opera dantesca.

Nell'immediato seguito, e per le prime due tavole del fumetto, il racconto prosegue in un modo che pare tradire la novità di questa apertura 'in costume'. Accade infatti che Topolino e Pippo, pur travestiti da Dante e Virgilio, paiono avviarsi secondo i consueti schemi narrativi delle storie Disney. I due incontrano prima Gambadilegno, che gli gioca un brutto tiro, e poi Minnie, tanto affettuosa quanto capricciosa, che inizialmente si complimenta per la loro bella iniziativa ma poi finisce per prendere Topolino a colpi di ombrello in testa. Tutti motivi narrativi ben noti ai lettori. Elementi di forte serialità, appunto. La cornice, per quanto breve, concilia il messaggio rassicurante della ripetizione seriale - 'lettore, stai leggendo un nuovo episodio delle storie di Topolino and friends che già conosci e apprezzi' - con la novità del travestimento, che prelude invece all'adattamento intersemiotico.

3. Serialità e intertestualità si intrecciano nel modo in cui i ruoli di Dante e Virgilio vengono ritagliati su Topolino e Pippo. Osserviamo i due protagonisti in una, per così dire, 'foto di scena' (Fig. 2).

Il travestimento è, come si è già detto, il più evidente indice dell'adattamento intertestuale, ed è dunque il rapporto di intertestualità a prevalere in questo caso sulla serialità. Invece degli abiti consueti, Topolino e Pippo indossano il tunicone e la corona d'alloro, in un evidente richiamo all'iconografia à la Doré associata tradizionalmente alla Commedia. È un esempio di come, sul versante dell'intertestualità, convergano sulla Parodia spunti provenienti dal complesso della tradizione della Commedia, oltre la lettera del testo dantesco. 


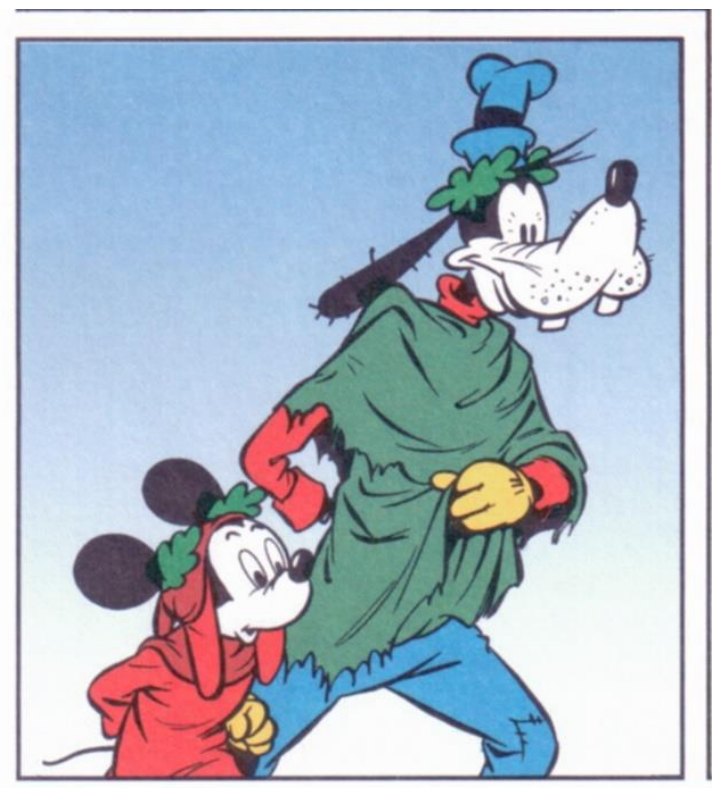

Figura 2

In linea con le attese del lettore seriale è invece la scelta di assegnare a Topolino il ruolo del protagonista. Topolino è infatti, ancora oggi, il personaggio più rappresentativo dell'universo disneyano, il suo marchio, e, in quell'epoca ancor più di ora, il protagonista usuale di tutte le storie Disney di una certa consistenza e impegno autoriale.

Secondo una logica analoga, una volta assegnato a Topolino il ruolo di Dante, spetta a Pippo 'di conseguenza', essendo la sua naturale spalla comica, il ruolo di Virgilio. L'assegnazione delle parti segue dunque una relazione preesistente tra Topolino e Pippo e ciò accade nonostante le caratteristiche della coppia disneyana si discostino, e non di poco, dai valori che sono invece propri della coppia Dante-Virgilio nella Commedia. Il vincolo di serialità e quello di intertestualità sono in questo caso divergenti: discende da questo semplice meccanismo una quota considerevole di effetto parodico.

Le caratteristiche di Pippo, così svitato e poco connesso con la realtà, rovesciano completamente il profilo della guida virgiliana. Il suo personaggio è una guida che non conosce il cammino, di poca acutezza e di scarso o nullo aiuto, al punto che non raramente è piuttosto Topolino a doverlo soccorrere e 
a vigilare sulla riuscita del viaggio, invece che il contrario. La fantasiosa varietà degli imprevisti nei quali ciò si verifica moltiplica l'effetto comico di questo rovesciamento, dando corpo a un insistito controcanto parodico dei numerosi luoghi della Commedia nei quali Virgilio opera in soccorso di Dante e ne sostiene il cammino. L'illustrazione riporta alcuni esempi (Fig. 3): è Topolino a riparare ingegnosamente la bicicletta di cui si servono lungo il cammino, sostituendoo allo pneumatico uno sbalordito serpente (Fig. 3a); sempre lui a convertire le Furie in due motori a reazione da montare sopra a Dumbo (Fig. 3b); ancora lui a salvare il compagno da Cerbero (Fig. 3c) o infine a impedire che cada nel tranello di un fosso di pece bollente che pare cioccolata (Fig. 3d).

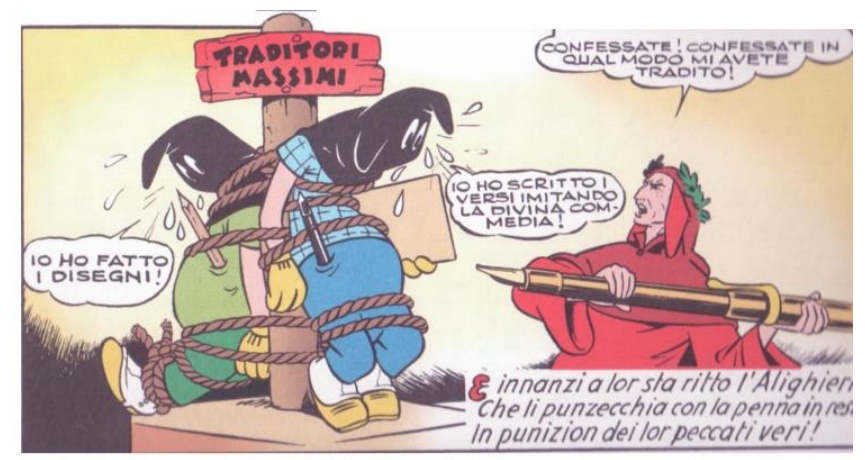

Figura 3

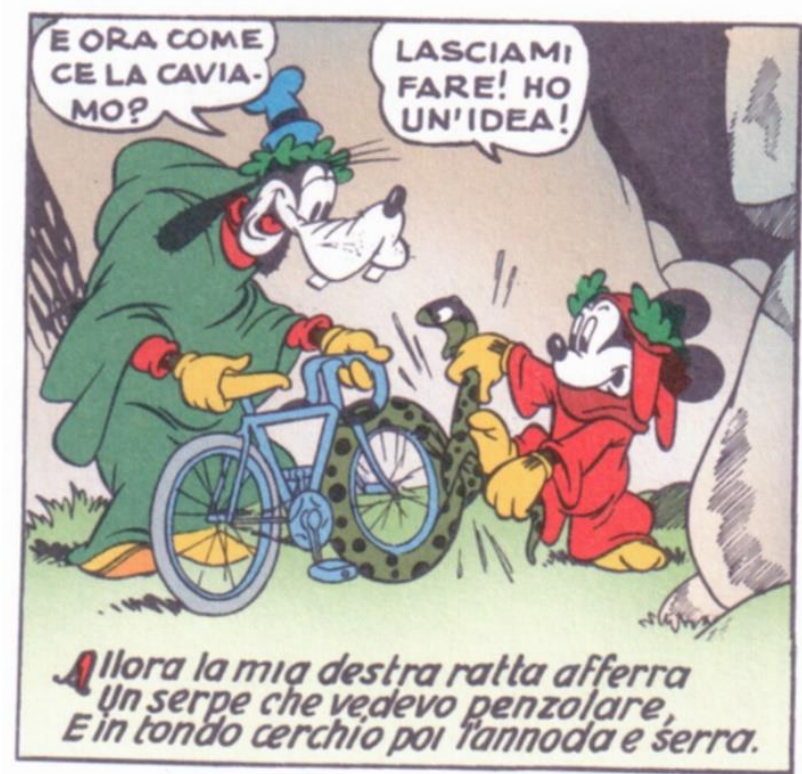




\section{Figura 3a}

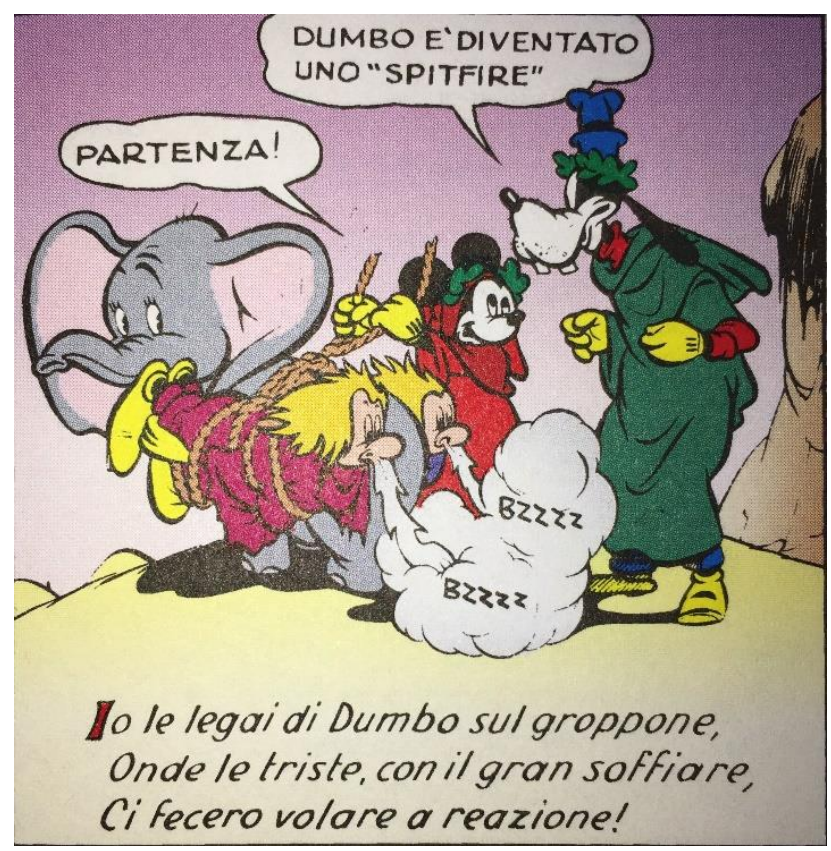

Figura 3b

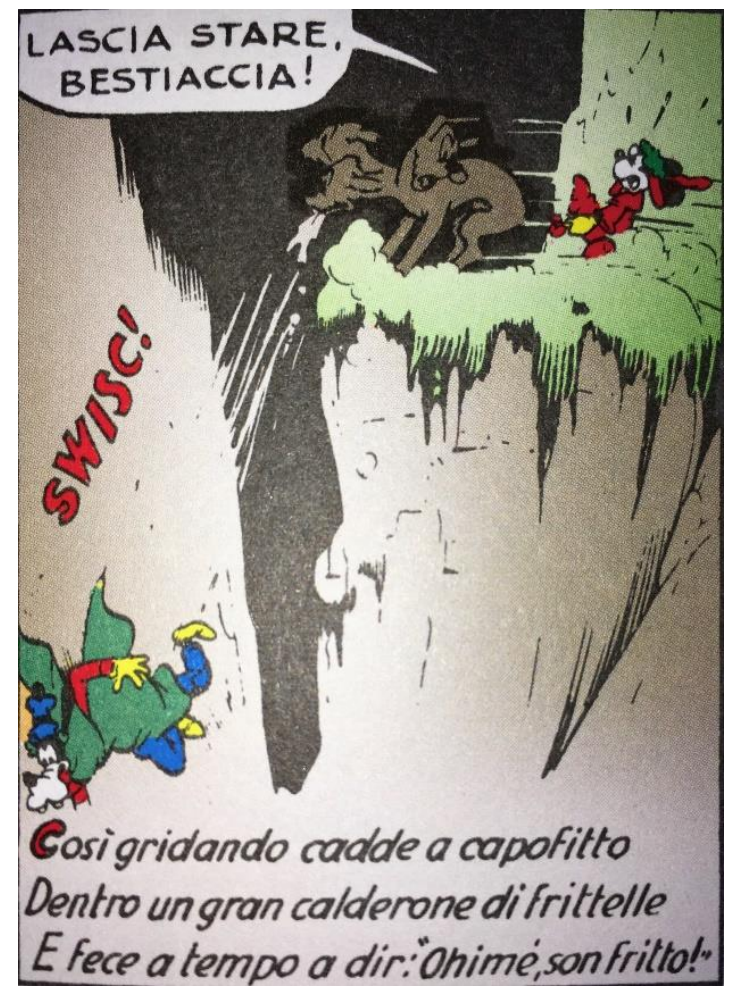

Figura 3c 


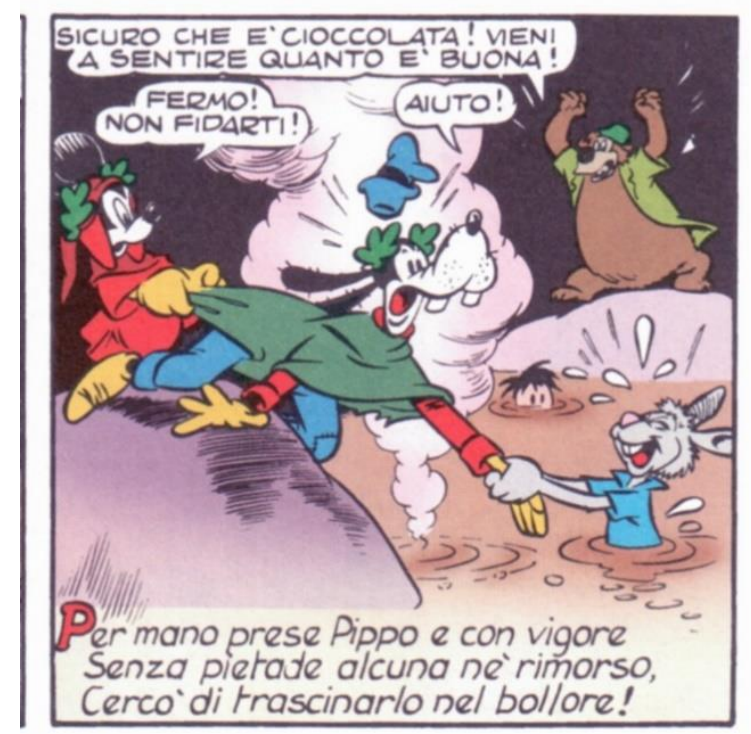

Figura 3d

4. L'assegnazione del ruolo di Dante a Topolino non segue soltanto la logica della serialità, dalla quale discende che inderogabilmente gli spetti il ruolo di protagonista. Il personaggio di Topolino, infatti, presenta di suo più di un elemento di rassomiglianza con il protagonista della Commedia. Sono entrambi, Dante e Topolino, campioni di caparbietà e forza d'animo, animati dal senso di un'inderogabile missione etica che li riguarda in prima persona e che orienta il loro cammino e le loro azioni. Ripetizione seriale e dialogo intertestuale paiono dunque, sotto questo aspetto, potersi allineare e convergere. Anche in questo caso, però, l'elemento disneyano finisce per prevalere su quello letterario. Avviene con una strategia articolata, che comporta per certi versi una semplificazione del testo dantesco e per altri un'espansione sul piano tematico e narrativo.

Topolino è in sostanza un brillante e generoso risolutore di problemi. Un eroe moralmente irreprensibile - a differenza per esempio di Paperino - e pragmaticamente votato alla concreta realizzazione del Bene. La sua intraprendenza, le sue doti, la sua bontà, conducono inevitabilmente le sue avven- 
ture seriali al lieto fine. Sulla base di queste premesse, il suo viaggio in Inferno non si può configurare, com'era invece quello dantesco, come un viaggio nelle manifestazioni più nefaste e irredimibili della realtà umana, accolte nell'affresco dantesco in quanto parti anch'esse dell'universale disegno divino. Più modestamente, il viaggio di Topolino sarà una vicenda nella quale il suo destino ingenuamente pragmatico può e deve compiersi una volta di più.

'Modestamente', tuttavia, non è l'avverbio più giusto. L'innovazione introdotta nella parodia affinché la vocazione risolutrice e operativa di Topolino abbia modo di manifestarsi è, infatti, molto audace. Per dirla in parole povere, la forza di Topolino è tale da permettergli di ottenere nientemeno che la salvezza dei dannati dell'Inferno. I suoi modi, la sua dedizione un po' spiccia, arrivano a qualcosa di assolutamente impensabile nella Divina Commedia, dove la dannazione degli abitanti infernali è eterna e immutabile e il tema della salvezza è esclusivo appannaggio del Purgatorio e del Paradiso. Nella parodia, invece, la pena si riduce a un circostanza spiacevole, ma risolvibile grazie all'intervento dell'eroe. Una punizione che è possibile riscattare con una semplice buona azione e semplicemente pentendosi.

Nel corso del racconto, un buon numero di incontri tra Topolino e i dannati si risolvono nella salvezza di questi ultimi. Topolino si interessa, osserva, consiglia, persuade al pentimento, intercede. Nell'elenco di coloro che via via beneficiano della sua azione salvifica troviamo i golosi interpretati Qui Quo Qua; poi i discoli pinocchieschi del paese dei balocchi; ancora, il suo amico-nemico Paperino; e infine, nel gran finale del fumetto, gli stessi autori della parodia.

5. Gli ultimi due fra gli episodi appena citati sono i più significativi. Paperino, in particolare, ha nella parodia un ruolo di assoluto primo piano per l'elaborazione narrativa del tema della salvezza. Il primo aspetto a 
caratterizzare la sua parte è il fatto che egli è l'unico personaggio della parodia a presentarsi ripetutamente nel corso del viaggio di Topolino e Pippo, intento ogni volta, unico antagonista dell'eroe, a ostacolarne il cammino. La sua persecuzione comincia fra le tombe della città di Dite, quando all'improvviso esce da un sarcofago per rinfacciare minacciosamente a Topolino il suo privilegio di viaggiatore infernale: "Ehi tu raccomandato di ferro! Prendi il mio posto e cedimi il tuo!" (Fig. 4a). Una seconda volta, compare nella formazione 'brasiliana' dei Tre Caballeros, con José Carioca e Panchito Pistoles, e aggredisce senza apparente motivazione Topolino, benché questi l'avesse appena salvato dal precipizio in cui rischiava di cadere (Fig. 4b). Passano poche pagine appena e ricompare incarnato in una delle tre Arpie (Fig. 4c) costringendo Topolino e Pippo a una precipitosa fuga. Di segno almeno parzialmente diverso la sua comparsa successiva, un piccolo cammeo all'interno dell'episodio a tema pinocchiesco: qui Paperino è tra i bambini che vengono perdonati e condotti in Paradiso, per quanto la sua promessa di pentimento non suoni poi così affidabile: “Anch’io sarò sempre buono (almeno, lo spero...)!" (fig. 4d) e lasci presagire le complicazioni a venire.

Complicazioni che puntualmente arrivano in una delle scene migliori del fumetto. L'episodio corrisponde a quello di Ulisse, come dimostra la grande fiamma biforcuta che campeggia al centro della tavola (Fig. 5). I due corni della fiamma non corrispondono qui però a Ulisse e Diomede. Essi rappresentano invece icasticamente l'incostanza caratteriale di Paperino, il suo oscillare umorale tra irascibilità e dolcezza, superbia e generosità, indolenza e lealtà. Con le parole in versi di Topolino che accompagnano l'episodio:

\footnotetext{
La prima dolcemente mi saluta Ma l'altra, con linguaggio da facchino,

Minacce e insulti torvamente sputa!

Perché sempre così fa Paperino

Talvolta è dispettoso e invelenito,
} 
Talvolta è dolce come un cherubino!

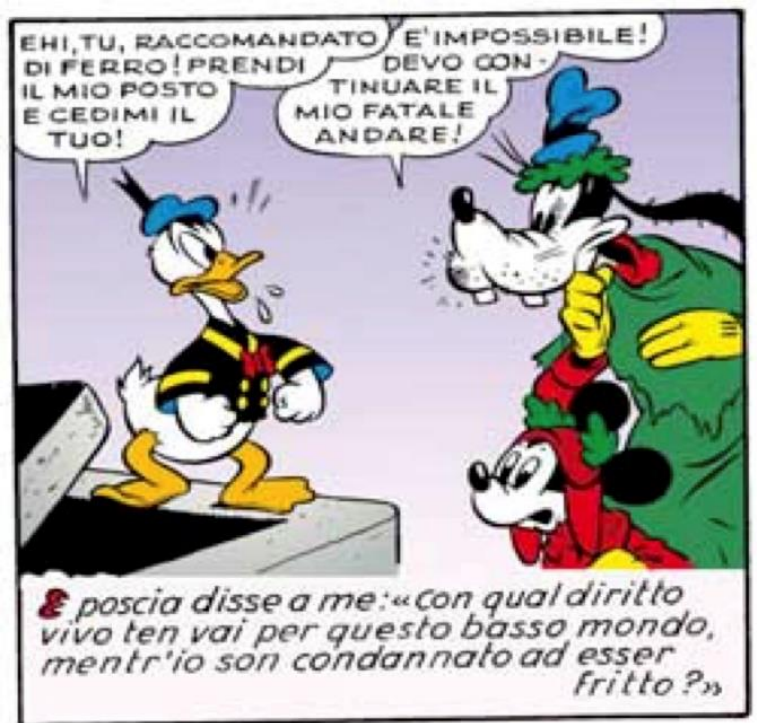

Figura 4a

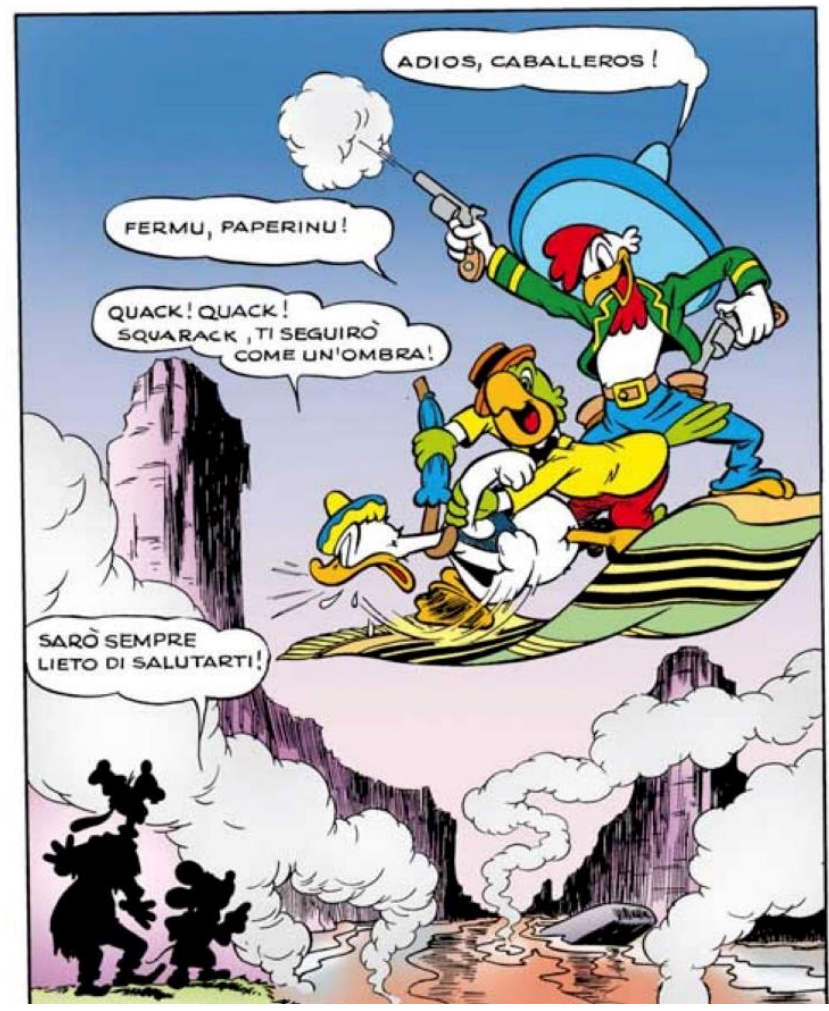

Figura 4b 


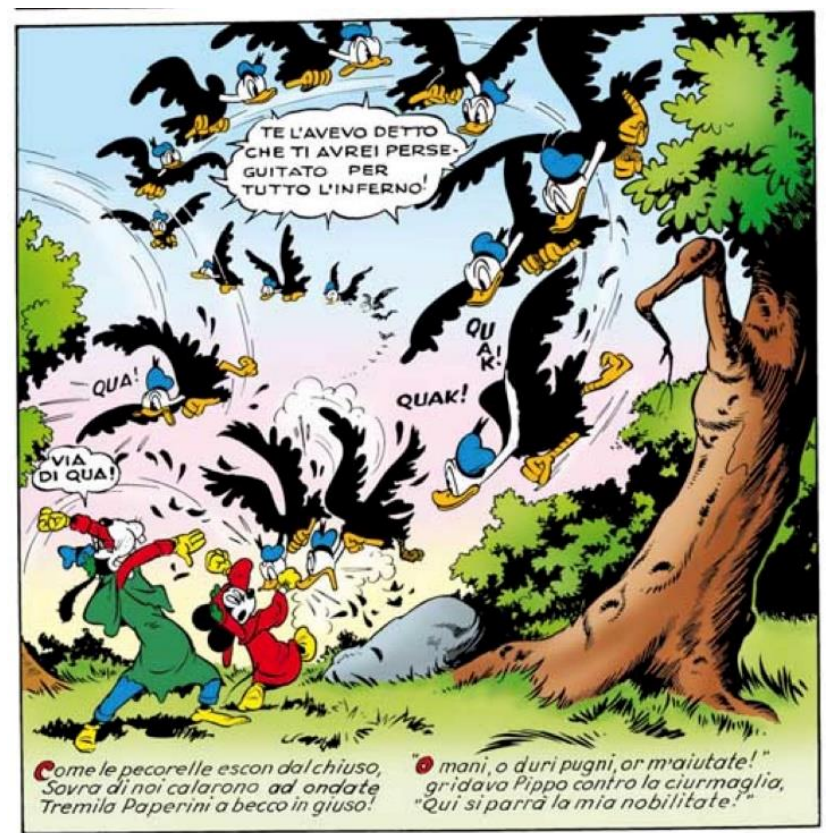

Figura 4c

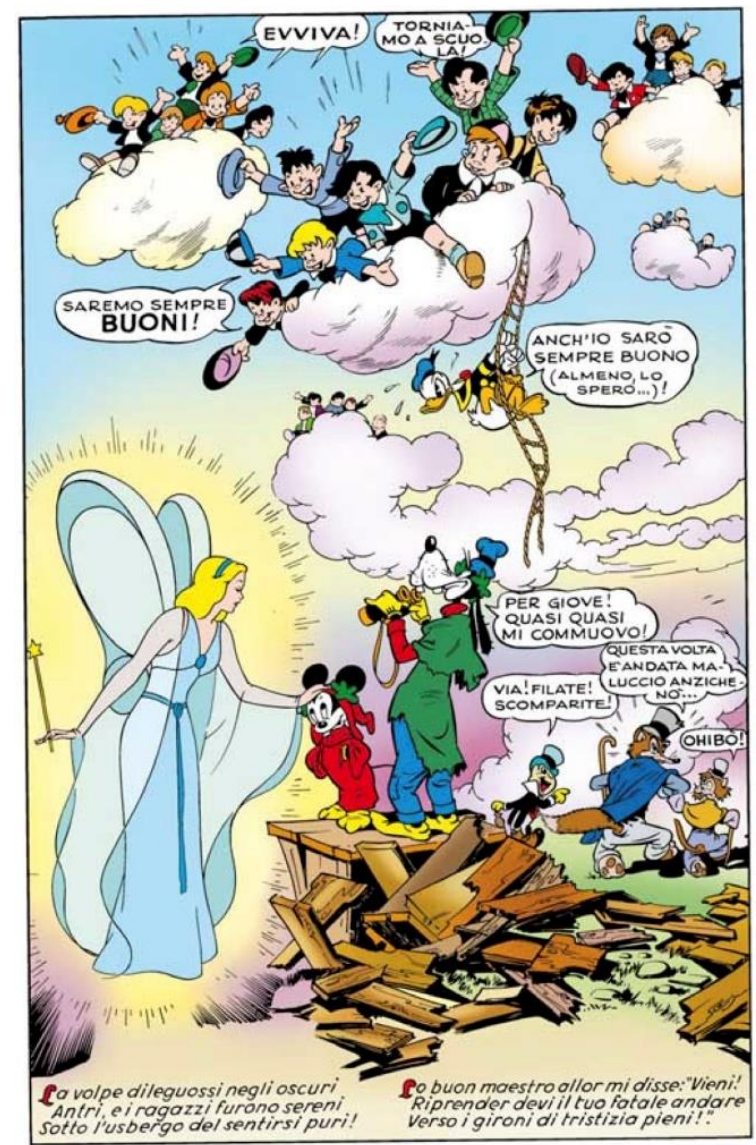

Figura 4d 


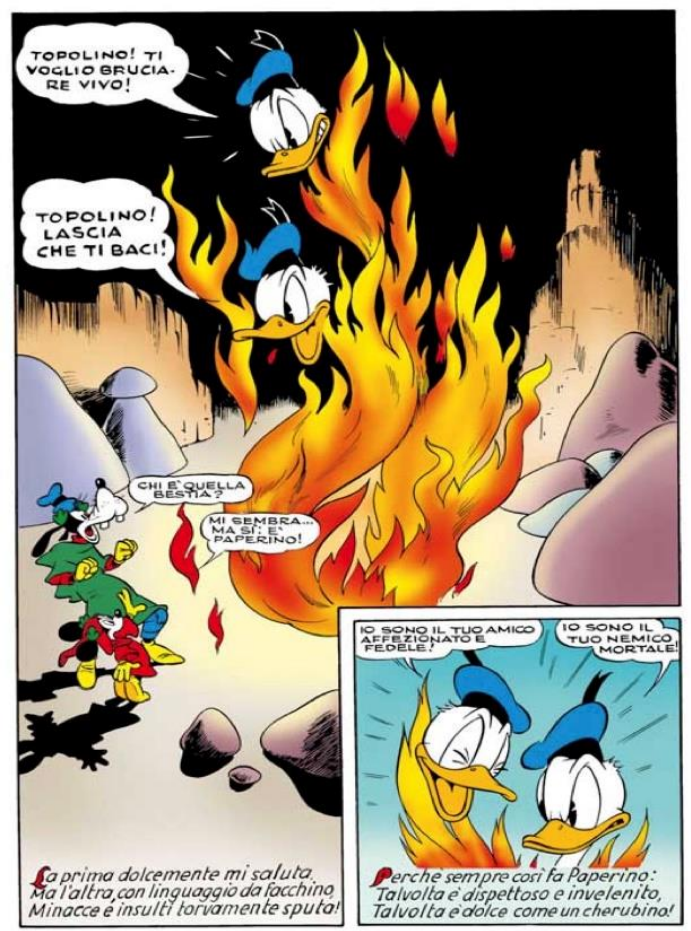

Figura 5

Compresa la situazione, Topolino non tarda ad entrare in azione: sicuro del prevalere della parte buona su quella cattiva, con l'aiuto di Pippo e di due provvidenziali estintori, spegne le framme e riconquista Paperino, integro e rappacificato, alla sua amicizia.

La salvezza di Paperino è l'episodio archetipico della capacità di Topolino/Dante di operare attivamente per la salvezza dei dannati, e marca in un aspetto decisivo della storia il prevalere della serialità sull'intertestualità. La rielaborazione dei temi dell'espiazione delle proprie colpe, del pentimento e della salvezza costituisce la più rilevante innovazione ideologica condotta nella parodia disneyana, ben al di là delle sue apparenze di innocua e infantile carnevalata. Si può discutere se in una valutazione critica dell'Inferno di Topolino debba prevalere il riconoscimento dell'importanza pedagogica di concetti quali la redimibilità della colpa e l'idea che un giusto premio segua sempre una giusta punizione e un sincero pentimento; oppure se non si debba invece guardare con sospetto, tanto più in un testo rivolto a un pubblico di giovani 
lettori, alla riproposizione di uno schema narrativo centrato su un'etica un po' superficiale e benpensante della 'buona azione'. La questione è aperta.

6. Un ultimo luogo testuale da tenere in considerazione in merito anche a questi interrogativi è il finale della parodia, che costituisce una sorta di manifesto implicito dell'intero corpus delle parodie Disney - ancora a venire all'epoca dell'Inferno di Topolino. Nel fumetto, come nell'originale, l'ultima tappa del viaggio infernale è l'incontro con i traditori massimi. Nel poema dantesco, Lucifero infieriva sulle anime dannate di Bruto, Cassio e Giuda; Topolino e Pippo si trovano invece di fronte a una analoga scena di punizione esemplare (Fig. 6), nella quale però Dante-autore - in persona, ovvero non interpretato da Topolino - si accanisce contro due uomini incappucciati e legati a un palo, che sotto tortura ammettono di essere gli autori dei testi (Guido Martina) e dei disegni (Angelo Bioletto). Con la scelta sorprendente - ma in fondo presente anche nella Commedia - di includere nel racconto i suoi stessi autori, la parodia vira clamorosamente in direzione metatestuale e metaletteraria.

La scena è densa di particolari evocativi - come un'allegoria dantesca, volendo. Dante-autore posa una mano su un mastodontico e paludato tomo della Divina Commedia, simbolo della sacralità dell'opera canonica, e tiene nell'altra, usandola come arma, una gigantesca penna stilografica, simbolo del suo privilegio autoriale. Tutti gli elementi del sistema letterario messi in gioco nell'adattamento sono qui portati sulla scena: gli autori e i lettori della parodia, i personaggi seriali che l'hanno impersonata, l'opera letteraria di partenza e il suo arcicanonico autore. Per il valore simbolico degli oggetti, dei personaggi e delle azioni raffigurate - la disapprovazione, la punizione, il pentimento, il perdono - il finale è in grado di svolgere in forma allusiva una discussione non banale e anzi decisamente problematica e articolata. 


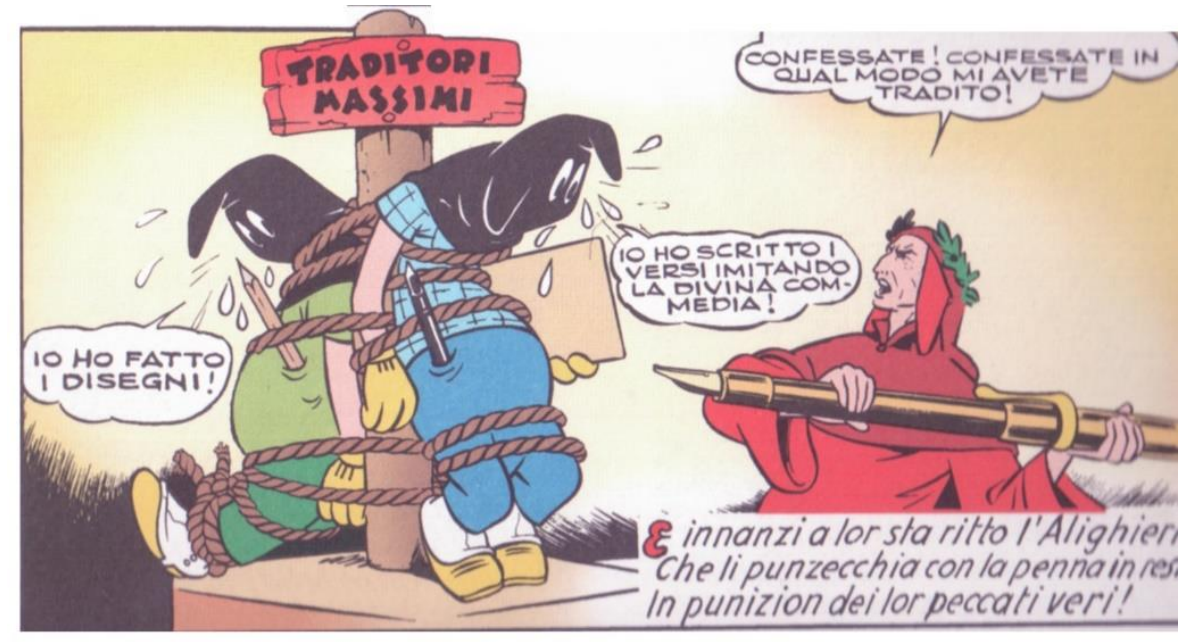

Figura 6

Il tema in ballo è l'effettivo valore artistico e culturale della parodia Disney, nell'ambito di una riflessione più ampia su quali siano le forme opportune per tramandare un'opera canonica quale la Commedia. Anche gli autori della parodia saranno infine perdonati e salvati. A risolvere la situazione sarà, ancora una volta, Topolino (Fig. 7). Senza alcun timore reverenziale, egli interviene per difenderli dall'accusa di aver 'mancato di rispetto' alla Divina Commedia, portando a loro favore l'argomento della funzionalità della parodia nel tramandare ai più giovani i valori fondamentali della Commedia. Il divertimento che provano i lettori del fumetto ("Non li senti gridare che davvero/Si sono divertiti come pazzi?..”), spiega Topolino, è non solo onesto, ma benefico per la tutela dei valori culturali e persino patriottici che la Commedia incarna. 

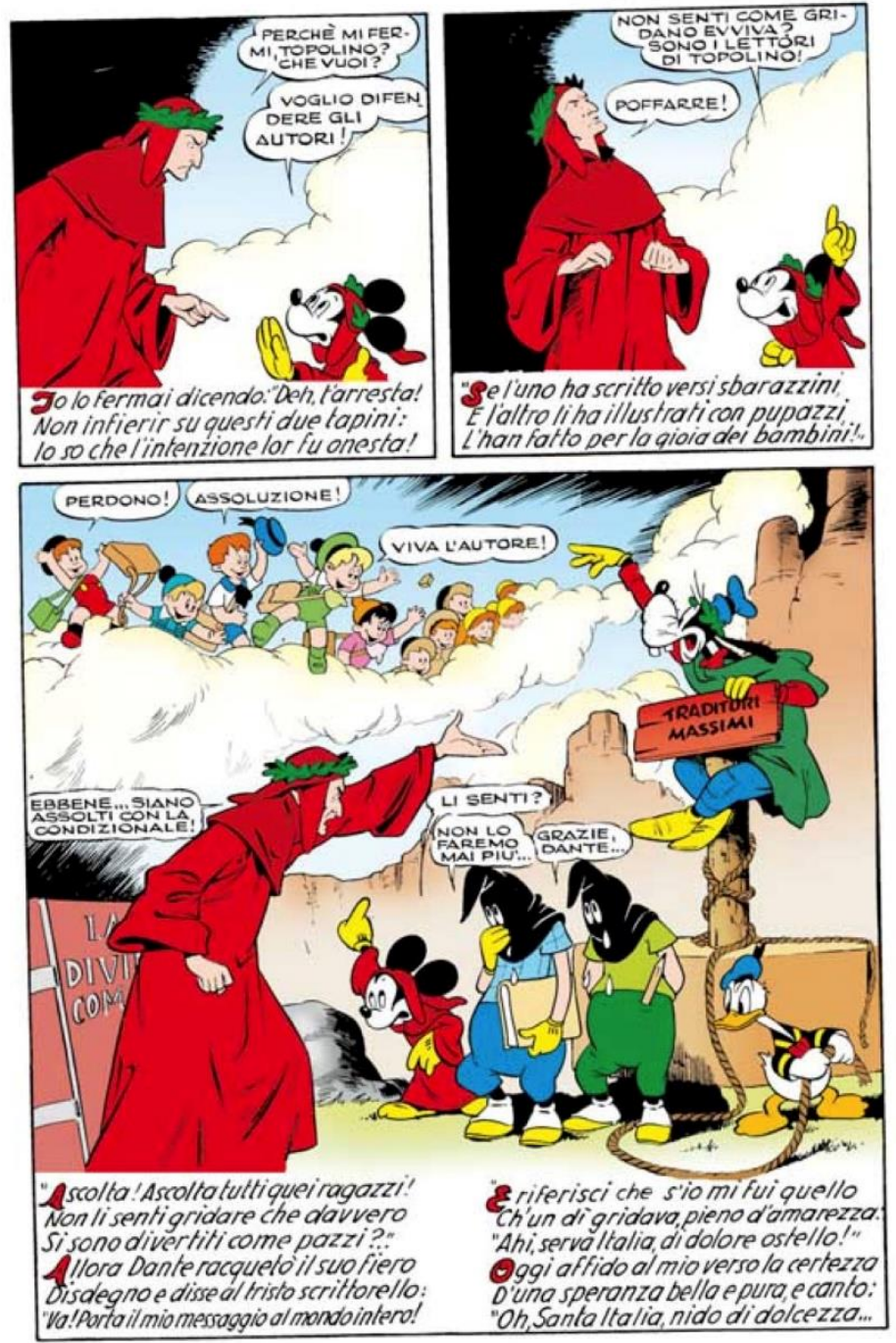

Figura 7

Più che gli argomenti addotti, in fondo non particolarmente originali, merita attenzione il gesto operato da Topolino: il suo coraggioso, o impertinente, 'prendere parola' per mediare tra istanze diverse del sistema letterario, tra letteratura e fumetto, e tra tradizione e innovazione, autorialità e libera rielaborazione. In linea con la sua natura seriale di generoso ed efficace risolutore di problemi e conflitti, e di indomito protagonista. Fuori metafora, si può leggere in questo gesto di Topolino una rivendicazione dell'importanza culturale del fumetto e dei personaggi seriali - o quantomeno di quelli disneyani. Essi partecipano attivamente e con un ruolo di primo piano, con 
forme proprie e risultati di rilievo, a un sistema culturale attraversato dalle tensioni che sorgono attorno a dinamiche complesse di dignità artistica, prestigio culturale, canonizzazione, tradizione.

\section{Considerazioni finali}

La peculiarità delle Grandi Parodie Disney dei classici della letteratura si basa su un procedimento adattivo capace di coniugare in modo originale intertestualità e serialità. La prevalenza di quest'ultima permette in primo luogo l'immediata riconoscibilità delle Parodie Disney, ma soprattutto libera il potenziale creativo della rielaborazione disneyana, secondo una gamma di procedimenti che vanno dalla semplificazione narrativa, alla soppressione, all'espansione tematica.

Nel caso dell'Inferno di Topolino le principali innovazioni sul piano tematico-ideologico riguardano, in linea con i valori identitari seriali dei personaggi disneyani, una forte attenuazione della tematica dantesca della condanna infernale. Ad essa corrisponde nel fumetto il refrain pedagogico di un perdono sempre possibile, per effetto del buon operare e del pentimento. Le parti più scopertamente metaletterarie del racconto a fumetti, e in particolare il finale, dimostrano l'alto grado di consapevolezza con il quale questa forma ibrida di produzione seriale rivendica un proprio ruolo entro le complesse dinamiche dell'intertestualità, del prestigio letterario e della tradizione culturale. 


\section{Bibliografia}

ARGIOLAS, Pier Paolo; CANNAS, Andrea; DISTEFANO, Giovanni Vito; GUGLIELMI, Marina. Le Grandi Parodie Disney, ovvero I Classici fra le nuvole. Roma: Nicola Pesce Editore, 2013.

ARNALDI, Valeria. Mickey Mouse: Mito e icona del personaggio cult di Walt Disney. Roma: Ultra, 2018.

BECATTINI, Alberto; BOSCHI, Luca; GORI, Leonardo; SANI, Andrea. I Disney italiani. Roma: Nicola Pesce Editore, Roma, 2012.

BERNARDELLI, Andrea. Eco e le forme della narrazione seriale: alcuni spunti per una discussione. Forme, strategie e mutazioni del racconto seriale, a cura di A. Bernardelli, E. Federici, G. Rossini, Between. VI.11, 2016, disponibile in http://www.betweenjournal.it.

ECO, Umberto. Tipologia della ripetizione. In Sugli specchi e altri saggi. Milano: Bompiani, 1985, 125-146.

FAETI, Antonio. In trappola col topo: una lettura di Mickey Mouse. Torino: Einaudi, 1986.

FOSSATI, Franco. Topolino: storia del topo più famoso del mondo. Milano: Gammalibri, 1980.

MARTINA, Guido. Le più belle storie disneyane scritte da Guido Martina. Milano: The Walt Disney Company Italia, 2003.

TOSTI, Andrea. Topolino e il fumetto Disney italiano: storia, fasti, declino e nuove prospettive. Latina: Tunué, 2011.

$1 \quad$ Cfr. Argiolas, Cannas, Distefano, Guglielmi 2013.

Cfr. Arnaldi 2018; Faeti 1986; Fossati 1980.

Cfr. Cfr. almeno Eco 1985; Bernardelli 2016.

Tra le tante storie disneyane nelle quali si ritrova questo dispositivo sono esemplari la Storia di Marco Polo detta il Milione, 1982, sceneggiatura di Guido Martina, disegni di Romano Scarpa, o ancora il ciclo della compagnia Alambrah, Miseria e nobiltà (1993), Il visconte dimezzato (1993), Le furberie di Scapino (1994), sceneggiatura di Lello Arena e Francesco Artibani, disegni di Giorgio Cavazzano e Silvia Ziche.

5 Cfr. Martina 2003; Becattini, Boschi, Gori, Sani 2012.

6 Cfr. Martina 2003; Becattini, Boschi, Gori, Sani 2012. 